\title{
Anisotropic diffusion of high-energy cosmic-ray electrons in the Galaxy
}

\author{
Shoko Miyake ${ }^{* 1 \dagger}$ \\ ${ }^{1}$ National Institute of Technology, Ibaraki College, Ibaraki, Japan
}

Effect of an anisotropic spatial diffusion with respect to the direction of the interstellar magnetic field (ISMF) on the energy spectrum of the high-energy cosmic-ray electrons is numerically investigated. Our calculation is performed by using a fully three-dimensional stochastic method based on the stochastic differential equations equivalent to the Parker transport equation that describes the propagation of the galactic cosmic rays (GCRs). We consider that the GCRs originate from the supernova remnants (SNRs) including known nearby young SNRs. We calculate the energy spectrum of high-energy GCR electrons by considering an anisotropic diffusion in the ISMF. Our results of the energy spectrum of high-energy GCR electrons show that the flux in the TeV regions strongly changes depending on the model of the diffusion coefficient.

35th International Cosmic Ray Conference - ICRC2017

10-20 July, 2017

Bexco, Busan, Korea

\footnotetext{
* Speaker.

${ }^{\dagger}$ E-mail: miyakesk@ee.ibaraki-ct.ac.jp
} 
Table 1: Distance from the solar system of each known SNR, age of each known SNR, and the cut-off energy of the energy spectrum of GCRs at each known SNR considered in our calculation.

\begin{tabular}{cccc}
\hline SNRs & Distance $[\mathrm{kpc}]$ & Age $\left[10^{3} \mathrm{yr}\right]$ & $E_{\text {cut }}[\mathrm{TeV}]$ \\
\hline SN185 & 0.95 & 1.8 & 170 \\
S147 & 0.80 & 4.6 & 63 \\
HB21 & 0.80 & 19 & 14 \\
G65.3+5.7 & 0.80 & 20 & 13 \\
Cygnus Loop & 0.44 & 20 & 13 \\
Vela & 0.30 & 11 & 25 \\
Monogem & 0.30 & 86 & 2.8 \\
Loop 1 & 0.17 & 200 & 1.2 \\
Geminga & 0.4 & 340 & 0.67 \\
\hline
\end{tabular}

\section{Introduction}

The most likely origins of high-energy galactic cosmic-ray electrons are localized in nearby young SNRs because of the large energy losses of the electrons by the inverse Compton scattering and the synchrotron radiation during the propagation in the Galaxy. So it is expected that the energy spectrum of the GCR electrons in the TeV region that would be presented by CALET [W] and DAMPE [D] in the near future provides information on the mechanisms of the propagation of the GCR electrons and their origins. Here we numerically investigate the effects of an anisotropic spatial diffusion with respect to the direction of the ISMF on the energy spectrum of GCR electrons in the $\mathrm{TeV}$ region. Our calculation is based on the stochastic differential equations (SDEs) equivalent to the Parker transport equation that describes the propagation process of GCRs in the Galaxy. We consider an anisotropic diffusion of the GCRs in the ISMF proposed by Jansson and Farrar[[]]. We also assume that the GCRs originate in SNRs including known SNRs that are located within $1 \mathrm{kpc}$ of the solar system and with an age less than $4 \times 10^{5} \mathrm{yr}$. The details of our model for the propagation of the GCRs are described in section 2. In section 3, we discuss the details of our results including the energy spectra of $\mathrm{TeV}$ electrons.

\section{Models}

In this study, we numerically investigate the propagation of GCRs that are originated in SNRs in the Galaxy. The calculation is made by using a fully three-dimensional stochastic numerical method proposed by Miyake et al. [四]. In this method, the supernovae ( $\mathrm{SNe}$ ) is assumed to be occur discretely in both space and time. We consider that the acceleration of GCRs in SNRs continues uniformly in time up to $10^{5}$ years after the explosion of the parent $\mathrm{SN}$, though it is suggested from recent studies that the escape time of GCRs from SNRs depends on their energy (e.g. Ohira et al. [四]; Caprioli et al. [鴫; Drury [四]). The radius of SNRs at the age of $10^{5}$ years is assumed to be 30 pc by following the Sedov model where the values of $10^{51} \mathrm{ergs}, 10^{9} \mathrm{~cm} \mathrm{sec}^{-1}$, and 1 proton $\mathrm{cm}^{-3}$ for the total explosion energy, the velocity of the shock wave, and the ambient matter density respectively are adopted. We also assume that the $\mathrm{SN}$ occurs at random both in time and space 


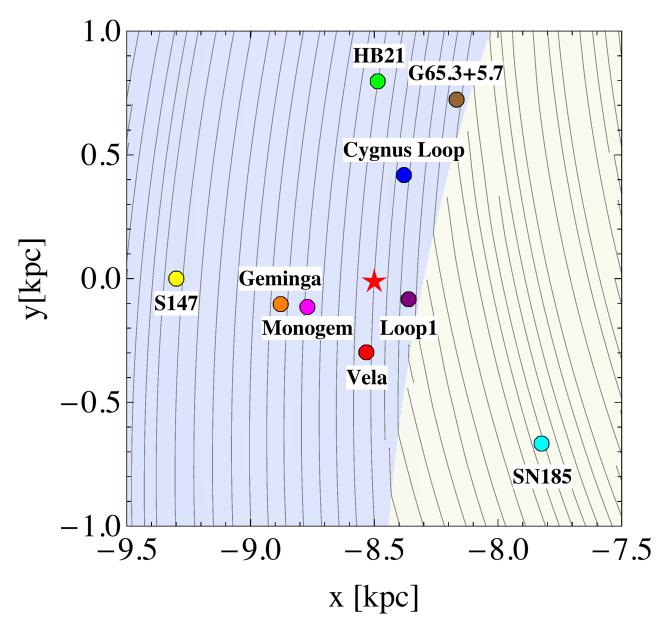

Figure 1: Two-dimensional position of the known nearby SNRs (circles) in the ISMF. Red star indicates the solar system.

within the radius of $20 \mathrm{kpc}$ from the center of the Galaxy, except for nearby young SNRs that are located within $1 \mathrm{kpc}$ of the solar system and with an age less than $4 \times 10^{5} \mathrm{yr}$ [[8]]. The frequency of occurrences of $\mathrm{SN}$ is assumed to be 3 times in 100 years in the Galaxy. The surface density of $\mathrm{SN}$ rate has a galactocentric radial dependence scaled to the molecular gas given by Williams \& McKee [Q], and has a Gaussian height distribution $P_{S N}=\left(2 \pi \alpha_{S N}^{2}\right)^{-1 / 2} \exp \left\{-z^{2} /\left(2 \alpha_{S N}^{2}\right)\right\} \mathrm{kpc}^{-1}$, where $\alpha_{S N}=0.070 \mathrm{kpc}$ (Boulares \& Cox [ए0]). We also consider the following known SNRs as the nearby young SNRs that are located within $1 \mathrm{kpc}$ of the solar system and with an age less than $4 \times 10^{5}$ yr [8]: SN185, S147, HB21, G65.3+5.7, Cygnus Loop, Vela, Monogem, Loop1, and Geminga. Table $\mathbb{W}$ shows the distance from the solar system of each known SNR, the age of each known SNR, and the cut-off energy of the energy spectrum of the GCRs at each known SNR. The positions of these known SNRs in the ISMF are shown in Figure $\square$.

We calculate the propagation of the GCRs in the Galaxy, in which an anisotropic spatial diffusion and the energy losses by synchrotron and inverse Compton processes of the GCR electrons are considered. The propagation of the GCRs are described by a coupled set of SDEs equivalent to the Parker's convection-diffusion equation, as follows.

$$
\begin{aligned}
& d \mathbf{r}=(\nabla \cdot \kappa) d t+\sum_{s} \sigma_{s} d W_{s}(t) \\
& d p=\frac{4}{3} \sigma_{\mathrm{T}} c \beta \gamma^{2} \frac{B^{2}}{8 \pi} d t+\frac{4}{3} \sigma_{\mathrm{T}} c \beta \gamma^{2} U_{\mathrm{ph}} d t, \text { (only for electrons) }
\end{aligned}
$$

where $\mathbf{r}$ and $p$ indicate the position and momentum of pseudo-particle respectively, $t$ is the time, $\kappa$ is the spatial diffusion coefficient tensor, $\sum_{s} \sigma_{s}^{\mu} \sigma_{s}^{v}=2 \kappa^{\mu \nu}, d W_{s}$ is the Wiener process given by the Gaussian distribution, $B$ is the magnitude of the ISMF, $\sigma_{\mathrm{T}}$ is the Thomson cross section, $\gamma$ is the Lorentz factor of electrons, and $U_{\mathrm{ph}}$ is the energy density of photons. We consider the following energy densities of photons that are different inside and outside the Galaxy.

$$
U_{\mathrm{ph}}=\left\{\begin{array}{l}
U_{\mathrm{star}}+U_{\mathrm{dust}}+U_{\mathrm{ph}}=0.45+0.20+0.26 \mathrm{eV} / \mathrm{cm}^{3} \quad \text { (inside the Galaxy) } \\
U_{\mathrm{ph}}=0.26 \mathrm{eV} / \mathrm{cm}^{3} \text { (outside the Galaxy) }
\end{array},\right.
$$



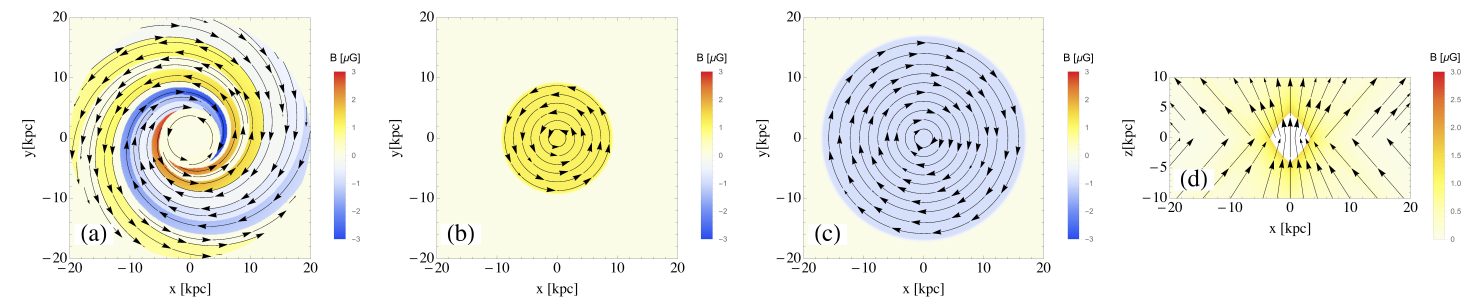

Figure 2: The large-scale regular GMF proposed by Jansson and Farrar [3]]. (a) disk component; (b) northern toroidal halo component at $z=1 \mathrm{kpc}$; (c) southern toroidal halo component at $z=-1 \mathrm{kpc}$; (d) out-of-plane component.

where $U_{\text {star }}, U_{\text {dust }}$, and $U_{\mathrm{CMB}}$ are the energy density of photons from the star, from the dust, and by the cosmic microwave background, respectively. The boundary of the Galaxy is assumed to be located on the radius of $20 \mathrm{kpc}$ from the center of the Galaxy and the height of $150 \mathrm{pc}$ from the galactic plane.

We presume the anisotropic spatial diffusion with respect to the direction of the ISMF that is proposed by Jansson and Farrar [B]. This ISMF consists of the disk component, the toroidal halo component, and the out-of-plane component as shown in Figure [ $[$. The mean free path of the particle is considered to be inversely proportional to the strengths of the ISMF. We adopted

$$
\begin{gathered}
\kappa_{\|}=\frac{1}{3} l_{\text {m.f.p. }} v \\
\frac{\kappa_{\perp}}{\kappa_{\|}}=\left\{\begin{array}{l}
1 \\
0.1
\end{array}\right. \\
l_{\text {m.f.p. }}=l_{0}\left(\frac{p}{1 \mathrm{GeV} / \mathrm{C}}\right)^{\delta}\left(\frac{B}{1 \mu \mathrm{G}}\right)^{-1},
\end{gathered}
$$

where $\kappa_{\|}$and $\kappa_{\perp}$ are the diffusion coefficients parallel and perpendicular to the magnetic field

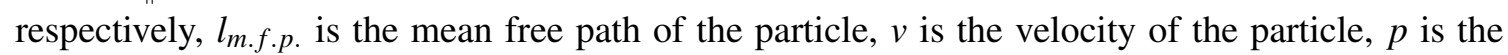
momentum of the particle, $B$ is the magnitude of ISMF, and $\delta$ is the power-law index related with the Alfvèn wave spectrum. We consider the values of $\delta$ changing from $\frac{1}{3}$ for Kolmogorov-type model to $\frac{1}{2}$ for Kraichnan-type model $\left(\delta=\frac{1}{3}, 0.40, \frac{1}{2}\right)$.

We determine the magnitude of $l_{0}$ for each model of $\delta$ so that our calculation reproduces the $\mathrm{B} / \mathrm{C}$ ratio at $1 \mathrm{GeV}$ observed by AMS- 02 [U]], as shown in Table $\mathbf{D}$. B/C ratio is calculated on the basis of the weighted slab method. To get the path length distribution that is needed for the weighted slab method, we calculate the path length $X$ simultaneously with the SDE as $d X=\rho v d t$ where $\rho$ is the density of the interstellar matter. The density $\rho$ is calculated by using the model of interstellar medium adopted by Higdon \& Lingenfelter [12]]. In this model, the interstellar medium consists of five major components, the molecular gas, the cold neutral gas, the warm neutral gas, the warm ionized gas, and the hot tenuous medium. The density of the molecular gas and the other components are given by Williams \& McKee [9] and Ferrière [1[3] respectively, as a function of the galactocentric radius and the height from the galactic plane. Only $\mathrm{C}, \mathrm{N}$, and $\mathrm{O}$ are considered to be the parents nuclei of B for simplicity. The source abundances of each parent nuclei were taken from Strong \& Moskalenko [14]. Relevant nuclear data were referred to Garcia-Munoz et al. [15], Webber et al. [ए]], and Ramaty et al [ए]]. 
Table 2: The absolute values of the mean free path of the particle $l_{0}$ for each model of $\delta$.

\begin{tabular}{cccc}
\hline & \multicolumn{3}{c}{$\delta$} \\
\cline { 2 - 4 }$\kappa_{\perp} / \kappa_{\|}$ & $1 / 3$ & 0.40 & $1 / 2$ \\
\hline 1.0 & 0.265 & 0.255 & 0.241 \\
0.1 & 0.846 & 0.794 & 0.758 \\
\hline
\end{tabular}

Note. All values in units of pc.

In our algorithm, first we generate a list of $\mathrm{SNe}$ which might have occurred during the last $5 \times 10^{8}$ yrs in our Galaxy so that their spatial position $\mathbf{r}_{\mathbf{S N}}$ and occurrence time $t_{S N}$ are randomly distributed to be consistent with the conditions as stated before. Next we start to follow the sample path $\mathbf{r}(t)$ with a certain energy by the SDE with an appropriate time step $\Delta t$. The path length for each time step can be calculated by $v \rho \Delta t$. Sample paths start at the solar system which is located at $(-8.5 \mathrm{kpc}, 0 \mathrm{kpc}, 0 \mathrm{kpc})$ for a galactocentric Cartesian coordinate system $(x, y, z)$, and then run backwards in time until they hit some active SNR centered at the parent SN on the prepared list where the acceleration of CRs is still going on. If the sample path $\mathbf{r}(t)$ at some time $t$ get into some SNR, in other words $\mathbf{r}(t)$ satisfies $\left|\mathbf{r}(t)-\mathbf{r}_{\mathbf{S N}}\right| \leq 30 \mathrm{pc}, t_{S N}-10^{5} \mathrm{yrs} \leq t \leq t_{S N}$, then we stop to calculate the sample path and we record the position, the arrival time, and the total path length, and then we restart the same procedure again for another particle. We follow the sample path up to $5 \times 10^{8}$ yrs while the particle fails to hit some active SNR. By this numerical experiment, we can simultaneously obtain the age distribution and the path length distribution for particles observed with various energies in the solar system.

\section{Result and Discussion}

We can obtain the spatial distribution of suspected source SNRs of GCRs that arrive at the solar system by our calculation. It is anticipated that the source SNRs of high-energy electrons have a tendency to be distributed in the direction along the magnetic field lines of the ISMF when the GCRs diffuse anisotropically, because the particles move faster along the magnetic fields lines than in the direction perpendicular to the field lines [2]]. Figure [ ] shows the resultant spatial distribution of suspected source SNRs of the high-energy electrons with energy of $2 \mathrm{TeV}$ at the solar system for two cases of anisotropy, $\kappa_{\perp} / \kappa_{\|}=1.0$ (left panel) and $\kappa_{\perp} / \kappa_{\|}=0.1$ (right panel). We can find that the distribution of the suspected source SNRs for the ratio of $\kappa_{\perp} / \kappa_{\|}=1.0$ is almost isotropic centered at the solar system, however in the case of $\kappa_{\perp} / \kappa_{\|}=0.1$ the suspected source SNRs are distributed along the field lines as anticipated.

We also be able to expect that the differences in the spatial distribution of suspected source SNRs for $\mathrm{TeV}$ electrons lead to the change of the spectral shape of the energy spectrum in $\mathrm{TeV}$ regions, because the most likely origins of high-energy galactic cosmic-ray electrons are localized in nearby young SNRs. Figure $\$$ shows our results of the energy spectra of GCR electrons above $100 \mathrm{GeV}$, which are obtained by considering various sets of the parameters of $\kappa_{\perp} / \kappa_{\|}$and $\delta$. We can find that the most $\mathrm{TeV}$ electrons originate form the nearby young SNRs and the energy spectrum strongly changes depending on the set of the parameters. The flux of $\mathrm{TeV}$ electrons for the case 

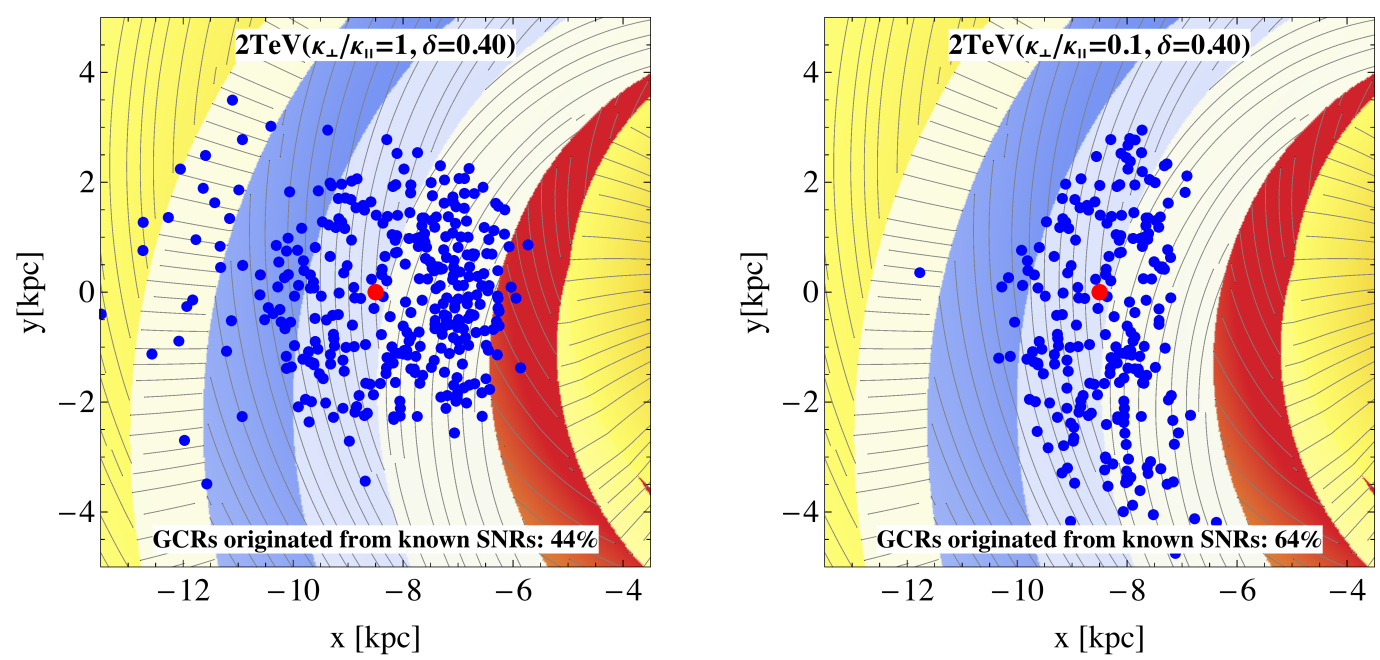

Figure 3: Spatial distribution of suspected source SNRs of GCR electrons with energy of $2 \mathrm{TeV}$ at the solar system for $\kappa_{\perp} / \kappa_{\|}=1.0$ (left panel) and $\kappa_{\perp} / \kappa_{\|}=0.1$ (right panel). $\delta=0.40$ is considered for both calculations. The red point and the blue points indicate the position of the solar system and the source SNRs of the sample particle arriving at the solar system, respectively.

of $\kappa_{\perp} / \kappa_{\|}=0.1$ is larger than that for the case of $\kappa_{\perp} / \kappa_{\|}=1.0$. This is because large amounts of $\mathrm{TeV}$ electrons originating from Vela and Cygnus Loop can reach the solar system by the diffusion process parallel to the magnetic field line.

Although the random component of the ISMF is not considered in this study, it is expect that the random component of the ISMF reduces the degree of the effect of the anisotropic diffusion on the energy spectrum of the GCRs. There also be still rooms for improvement in our model for the origin of the GCRs. It is suggested from recent studies that the escape time of GCRs from SNRs

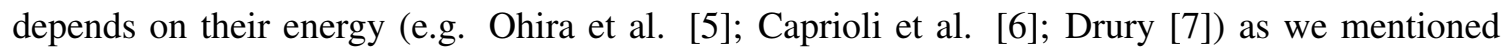
before. The contribution of pulsars to the local flux of the GCR electrons and positrons also be suggested from recent observations of gamma-ray such as Fermi-LAT (e.g. Gendelev et al. [[2]]). The impact of these observational facts that are not considered in this study on the energy spectrum of high-energy GCR electrons will be discussed in another paper.

\section{Acknowledgments}

We express our sincere thanks for the invaluable comments for this work of S. Yanagita, J. Nishimura, T. Kamae, J. W. Mitchel, K. Sakai, M. Sasaki, J. Krizmanic, A. Moiseev, and Y. Akaike. S.M.'s work is supported by JSPS KAKENHI Grant Number JP26800145.

\section{References}

[1] Y. Asaoka, Y. Akaike, Y. Komiya, R. Miyata, S. Torii, et al. Astrophart Phys. 91 (2017) 1-10.

[2] DAMPE Collaboration eprint arXiv:1706.08453 (2016).

[3] F. Jansson and G. R. Farrar Astrophys J. 757 (2012) 14.

[4] S. Miyake, H. Muraishi, and S. Yanagita Astronomy and Astrophys. 573 (2015). 

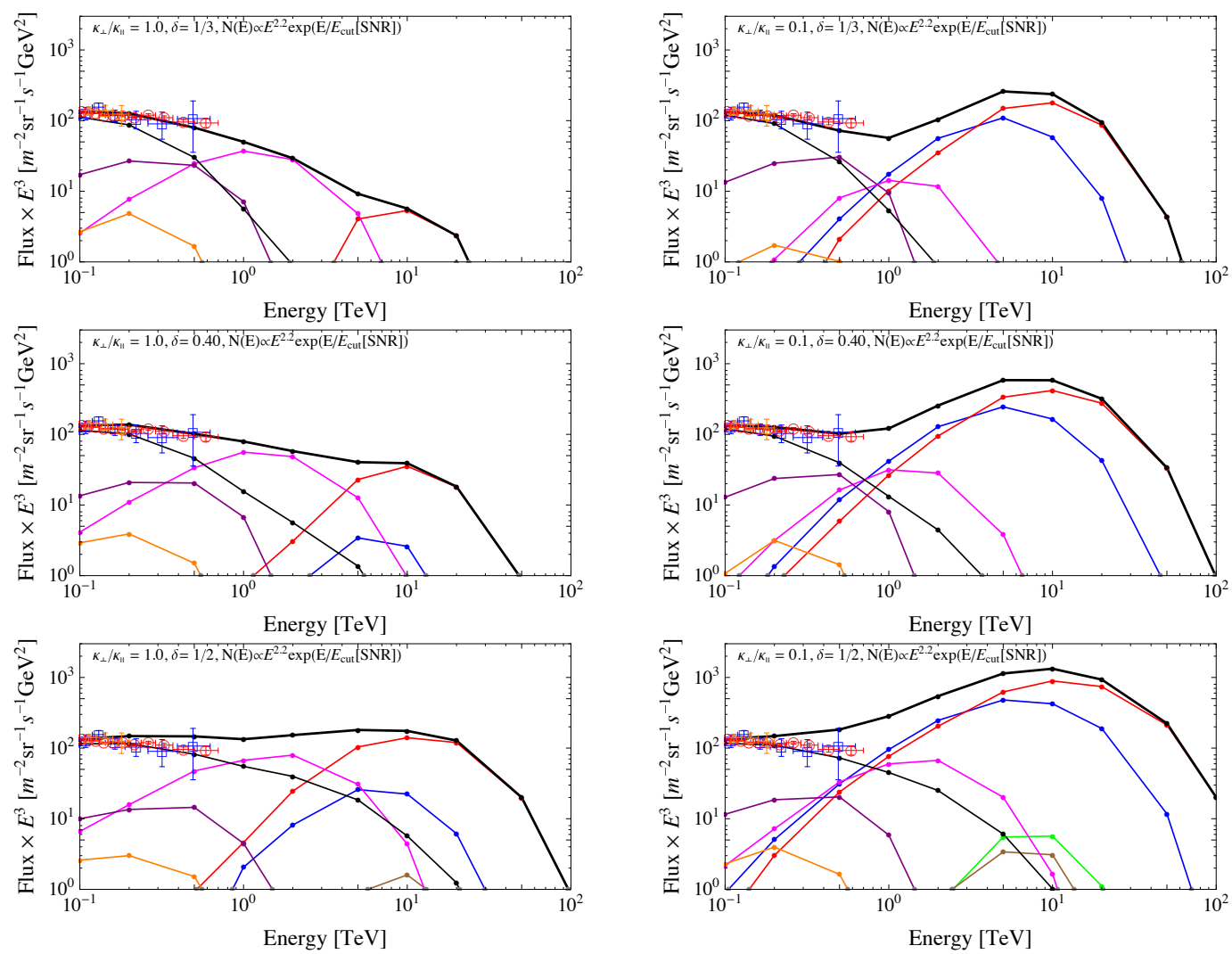

Figure 4: The energy spectra of the high-energy electrons. The solid lines except for the black lines indicate the energy spectra of electrons that are originated from nearby young SNRs assumed in this calculations: green line, for HB21; brown line, for G65.3+5.7; blue line, for Cygnus Loop; red line, for Vela; pink line, for Monogem; purple line, for Loop1; and orange line, for Geminga. Black thin line and thick line indicate the energy spectra of electrons that are originated from the other SNRs and the total energy spectra of all electrons, respectively. Red circles, blue squares, and orange triangles indicate the observations by AMS-02 [ए8], PAMELA [एप], and Fermi-Large Area Telescope (LAT) [ㅁ]]. The values of parameters considered in this calculation are shown in each figures.

[5] Y. Ohira, K. Murase, and R. Yamazaki Astronomy and Astrophys. 513 (2010) A17.

[6] D. Caprioli, E. Amato, and P. Blasi Astropart. Phys. 33 (2010) 160.

[7] L. O. Drury MNRAS 415 (2011) 1807.

[8] T. Kobayashi, Y. Komori, K. Yoshida, and J. Nishimura Astrophys. J 601 (2004) 340.

[9] J. P. Williams and C. F. McKee Astrophys. J. 476 (1997) 166.

[10] A. Boulares and D. P. Cox Astrophys. J. 365 (1990) 544.

[11] A. Oliva and A. Collaboration Proc. If 33rd ICRC (Rio de Janeiro) (2013).

[12] J. C. Higdon and R. E. Lingenfelter Astrophys. J. 582 (2003) 330.

[13] K. Ferrière Astrophys. J. 497 (1998) 759.

[14] A. W. Strong and I. V. Moskalenko Adv. Space Res. 717 (2001) 27.

[15] M. Garcia-Munoz, J. A. Simpson, T. G. Guzick, J. F. Wefel, and S. H. Margolis Astriphys J. Suppl. 64 (1987) 269. 
[16] W. R. Webber, A. Soutoul, J. C. Kish, and J. M. Rockstroh Astrophys. J. Suppl. 144 (2003) 153.

[17] R. Ramaty, B. Kozlovsky, R. E. Lingenfelter, and H. Reeves Astrophys. J. 488 (1997) 730.

[18] M. Aguilar, D. Aisa, A. Alvino, G. Ambrosi, K. Andeen, et al. Phys. Rev. Lett. 113 (2014) 121102.

[19] O. Adriani, G. C. Barbarino, G. A. B. R. Bellotti, M. Boezio, et al. Phys. Rev. Lett 106 (2011) 201101.

[20] M. Ackermann, M. Ajello, A. Allafort, W. B. Atwood, L. Baldini, et al. Phys. Rev. Lett. 108 (2012) 011103.

[21] S. Miyake and S. Yanagita Proc. of Science (ICRC2015) 491 (2015).

[22] L. Gendelev, S. Profumo, and M. Dormody Journal of Cosmology and Astropart. Physics 2 (2010) 016. 